\title{
Constraints on Neutrino Masses from Cosmological Observations
}

\section{B. Hoeneisen* ${ }^{\dagger}$}

Universidad San Francisco de Quito, Quito, Ecuador

E-mail: bhoeneisen@usfq.edu.ed

Fitting the predictions of the $\Lambda \mathrm{CDM}$ model to the Sachs-Wolfe effect, $\sigma_{8}$, the galaxy power spectrum $P_{\text {gal }}(k)$, fluctuations of galaxy counts in spheres of radii ranging from $16 / h$ to $128 / h \mathrm{Mpc}$, Baryon Acoustic Oscillation (BAO) measurements, and $h=0.678 \pm 0.009$, in various combinations, with free spectral index $n_{s}$, and free galaxy bias and galaxy bias slope, we obtain consistent measurements of the sum of neutrino masses $\sum m_{v}$. The results depend on $h$, so we have presented confidence contours in the $\left(\sum m_{v}, h\right)$ plane.

2nd World Summit: Exploring the Dark Side of the Universe 25-29 June, 2018

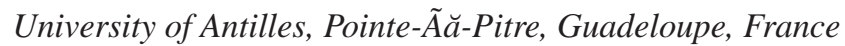

* Speaker.

${ }^{\dagger}$ I thank the organizers of the Guadeloupe Conference for the kind invitation. 


\section{Introduction}

The power spectrum of linear density perturbations $P(k)$ becomes suppressed at large wavenumber $k$ due to free-streaming of massive neutrinos that can not cluster on these small scales, and, more importantly, due to the slower growth of structure with massive neutrinos [何]. This suppression of $P(k)$ at large $k$ affects $\sigma_{8}$ that is sensitive to $\log _{10}\left(k /\left(h \mathrm{Mpc}^{-1}\right)\right)$ in the range -1.3 to -0.6 , while the Sachs-Wolfe effect, that is sensitive in the range -3.1 to -2.7 , is unsuppressed. It is therefore possible to measure neutrino masses by fitting the predictions of the $\Lambda \mathrm{CDM}$ model [2] to measurements of the Sachs-Wolfe effect and $\sigma_{8}$. Measurements of the galaxy power spectrum $P_{\text {gal }}(k)$ can also contribute to constrain neutrino masses if the bias $b$, defined by $P_{\text {gal }}(k)=b^{2} P(k)$, is understood. The Sachs-Wolfe effect of fluctuations of the Cosmic Microwave Background (CMB) provides a direct measurement of density fluctuations [2, 3]. The relative mass fluctuation $\sigma_{8}$ in randomly placed spheres of radius $r_{s}=8 / h \mathrm{Mpc}$ is measured with gravitational lensing and studies of rich galaxy clusters [ $[$ ].

To be specific, we consider three active neutrino eigenstates with nearly the same mass, so $\sum m_{v} \approx 3 m_{v}$. The suppression factor of $P(k)$ for large $k$ is $f\left(k, \sum m_{v}\right)=1-8 f_{v}$, where $f_{v}=\Omega_{v} / \Omega_{m}$ [1]. $\Omega_{m}$ is the total (dark plus baryonic plus neutrino) matter density today relative to the critical density, and includes the contribution $\Omega_{v}=h^{-2} \sum m_{v} / 93.04 \mathrm{eV}$ of neutrinos that are non-relativistic today.

In this note we outline the results of measurements of $\sum m_{v}$. For details we refer the reader to the talk at the Guadeloupe 2018 Conference [5], and to [6] and references therein.

\section{Measurement of neutrino masses with the Sachs-Wolfe effect and $\sigma_{8}$}

The $\Lambda$ CDM model prediction for $P(k)$ [⿰] spectral index $n_{s}$, and $\sum m_{v}$. We keep $n_{s}$ fixed. We vary the two parameters $N^{2}$ and $\sum m_{v}$ to minimize a $\chi^{2}$ with two terms corresponding to two observables: the Sachs-Wolfe effect that constrains $N^{2}$, and $\sigma_{8}$. We therefore have zero degrees of freedom. The result is a function of $h, \Omega_{m}$, and $n_{s}$, so we define $\delta h \equiv(h-0.678) / 0.009, \delta \Omega_{m} \equiv\left(\Omega_{m}-0.281\right) / 0.003$, and $\delta n \equiv\left(n_{s}-1\right) / 0.038$, and obtain

$$
\sum m_{v}=0.595+0.047 \cdot \delta h+0.226 \cdot \delta n+0.022 \cdot \delta \Omega_{m} \pm 0.225(\text { stat })_{-0.152}^{+0.484}(\text { syst }) \mathrm{eV} .
$$

\section{Test of scale invariance of the galaxy bias $b$}

We count galaxies in an array of $N_{s}=N_{x} \times N_{y}$ spheres of radii $r_{s}$, and obtain their mean $\bar{N}$, and their root-mean-square (rms). All spheres have their center at redshift $z=0.5$ to ensure the homogeneity of the galaxy selections. We compare $\sigma / \bar{N}$ obtained from galaxy counts, with the predicted relative mass fluctuation in the linear approximation corresponding to $P(k)$. The ratio of these two quantities is the bias $b$. This bias $b$ depends on $\sum m_{v}, h$, and $n_{s}$. We find that the galaxy bias $b$ is scale invariant, within the statistical uncertainties, if

$$
\sum m_{v}=0.939+0.035 \cdot \delta h+0.089 \cdot \delta n \pm 0.008 \mathrm{eV}
$$

else scale invariance is broken. Since scale invariance depends on $\sum m_{v}$ we allow $b$ to depend on $k$ in the following fits. 


\section{Measurement of neutrino masses with the Sachs-Wolfe effect, $\sigma_{8}$, and $P_{\text {gal }}(k)$}

We fit the Sachs-Wolfe effect, $\sigma_{8}, h=0.678 \pm 0.009$ [ $\bigoplus$, and the measurement of $P_{\text {gal }}(k)$ with galaxies in the Sloan Digital Sky Survey SDSS-III by the BOSS Collaboration [7, 8]. We allow the galaxy bias $b$ to depend on scale: $b \equiv b_{0}+b_{1} \log _{10}\left(k / h \mathrm{Mpc}^{-1}\right)$. Minimizing the $\chi^{2}$ with respect to $\sum m_{v}, N^{2}, n_{s}, h=0.678 \pm 0.009, b_{0}$, and $b_{1}$, we obtain

$$
\begin{aligned}
\sum m_{v} & =0.80 \pm 0.23 \mathrm{eV}, \\
N^{2} & =(1.88 \pm 0.39) \times 10^{-10}, \\
n_{s} & =1.064 \pm 0.068, \\
h & =0.676 \pm 0.011, \\
b_{0} & =2.35 \pm 0.36, \\
b_{1} & =0.229 \pm 0.094,
\end{aligned}
$$

with $\chi^{2}=27.8$ for 18 degrees of freedom. The uncertainties have been multiplied by $\sqrt{(27.8 / 18)}$. Confidence contours are presented in Fig. 11. Fixing $b_{1}=0$ obtains $\chi^{2}=36.3$, so including the scale dependence of $b$ is necessary. This measurement of $\sum m_{v}$ is interesting, but we do not use it in our final combination because of the high $\chi^{2}$ per degree of freedom (and the need for a better understanding of the galaxy bias).

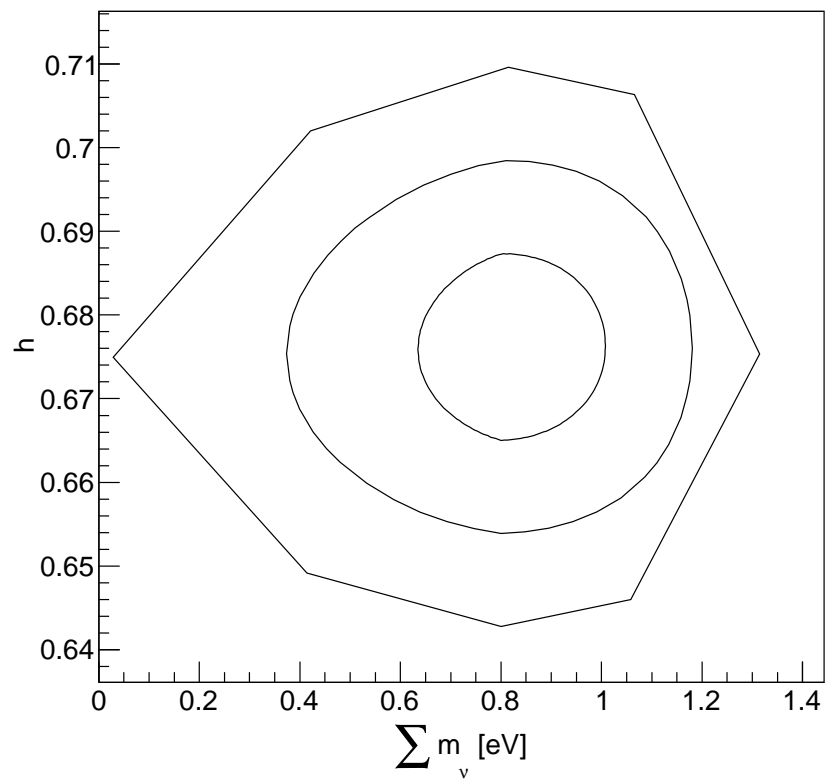

Figure 1: Contours corresponding to 1,2, and 3 standard deviations in the $\left(\sum m_{v}, h\right)$ plane, from SachsWolfe, $\sigma_{8}, h=0.678 \pm 0.009$, and $P_{\text {gal }}(k)$ measurements. Points on the contours have $\chi^{2}-\chi_{\min }^{2}=1,4$, and 9 , respectively, where $\chi^{2}$ has been minimized with respect to $N^{2}, n_{s}, b_{0}$, and $b_{1}$. 


\section{Measurement of neutrino masses with the Sachs-Wolfe effect, $\sigma_{8}$, and galaxy fluctuations}

We repeat the measurements of Section 1 but add 4 more experimental constraints: $\sigma / \bar{N}$ of SDSS DR14 [6, 8, 9] galaxy counts in spheres of radius $r_{s}=16 / h, 32 / h, 64 / h$, and $128 / h \mathrm{Mpc}$. We add two more parameters to be fit: $b_{0}$ and $b_{s}$ which define the bias $b=b_{0}-i_{s} b_{s}$, with $i_{s}=0,1,2,3$ for $r_{s}=16 / h, 32 / h, 64 / h$, and $128 / h \mathrm{Mpc}$, respectively. From the Sachs-Wolfe effect, $\sigma_{8}$, and the $4 \sigma / \bar{N}$ measurements we obtain

$$
\sum m_{v}=0.618+0.042 \cdot \delta h+0.206 \cdot \delta n+0.019 \cdot \delta \Omega_{m} \pm 0.209 \text { (stat) }{ }_{-0.139}^{+0.420}(\mathrm{syst}) \mathrm{eV},
$$

with $\chi^{2}=1.1$ for 2 degrees of freedom. The variables that minimize the $\chi^{2}$ are $\sum m_{v}, N^{2}, b_{0}$, and $b_{s}$. This result may be compared with (2.1).

\section{Combination with BAO}

In the companion talk and note in this Guadeloupe 2018 Conference [5] we obtained

$$
\sum m_{v}=0.711-0.335 \cdot \delta h+0.050 \cdot \delta b \pm 0.063 \mathrm{eV}
$$

where $\delta b \equiv\left(\Omega_{b} h^{2}-0.02226\right) / 0.00023$, from a study of Baryon Acoustic Oscillations (BAO) with SDSS DR13 galaxies and $\theta_{\mathrm{MC}}$ [8, 9, 10, 11]. We allow $\Omega_{b} h^{2}$ to vary by one standard deviation, i.e. $\delta b=0 \pm 1$ [4]. Combining with (5.1) we obtain

$$
\sum m_{v}=0.697-0.276 \cdot \delta h+0.032 \cdot \delta n+0.003 \cdot \delta \Omega_{m} \pm 0.075 \text { (stat) }{ }_{-0.028}^{+0.055}(\mathrm{syst}) \mathrm{eV}
$$

with $\chi^{2}=1.3$ for 3 degrees of freedom. Freeing $n_{s}$, and minimizing the $\chi^{2}$ with respect to $\sum m_{v}$, $N^{2}, n_{s}, h=0.678 \pm 0.009, b_{0}$, and $b_{s}$, we obtain

$$
\begin{aligned}
\sum m_{v} & =0.719 \pm 0.312(\text { stat })_{-0.028}^{+0.055}(\text { syst }) \mathrm{eV} \\
N^{2} & =(2.09 \pm 0.33) \times 10^{-10} \\
n_{s} & =1.021 \pm 0.075 \\
h & =0.678 \pm 0.008 \\
b_{0} & =1.751 \pm 0.060 \\
b_{s} & =-0.053 \pm 0.041
\end{aligned}
$$

with $\chi^{2}=1.1$ for 2 degrees of freedom. The uncertainty of $\sum m_{v}$ is dominated by the uncertainty of $h$, so we present confidence contours in the $\left(\sum m_{v}, h\right)$ plane in Figure 2 .

\section{Tensions}

Let us comment on Equations (6.1) and (5.1). Equation (6.1) is mainly determined by the precise measurement of the sound horizon angle $\theta_{\mathrm{MC}}$ by the Planck experiment, and by the assumption that the BAO wave stalls at redshift $z=z_{*}=1089.9 \pm 0.4$. Equation (6.1) tells us that $\left(\sum m_{v}, h\right)$ lies on the diagonal shown in Figure 2 (with some uncertainty from $\Omega_{b} h^{2}$ ). Equation 


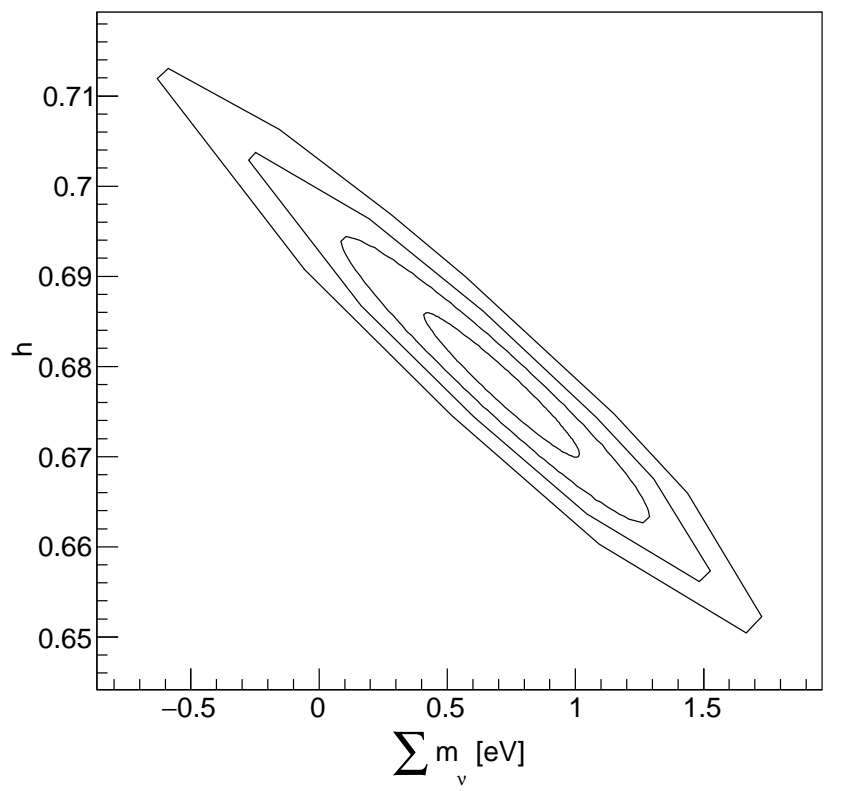

Figure 2: Contours corresponding to 1, 2, 3, and 4 standard deviations in the $\left(\sum m_{v}, h\right)$ plane, from SachsWolfe, $\sigma_{8}, 4 \sigma / \bar{N}$, BAO, and $h=0.678 \pm 0.009$ measurements. Points on the contours have $\chi^{2}-\chi_{\text {min }}^{2}=$ $1,4,9$, and 16, respectively, where $\chi^{2}$ has been minimized with respect to $N^{2}, n_{s}, b_{0}$, and $b_{s}$. The total uncertainty of $\sum m_{v}$ is dominated by the uncertainty of $h$. In this figure the systematic uncertainties presented in Equation (6.3) are not included.

(5.1) is a constraint mainly between $\sum m_{v}$ and $n_{s}$ with large uncertainties. To determine $\sum m_{v}$ we need as input a value for $h$ (or a value for $n_{s}$ ). In this article we have taken $h=0.678 \pm 0.009$ from [ [ however $h=0.688 \pm 0.009$ we obtain $\sum m_{v}=0.412 \pm 0.328 \mathrm{eV}$, and $n_{s}=0.960 \pm 0.073$. And if $h \approx 0.697$, we obtain $\sum m_{v} \approx 0 \mathrm{eV}$. Alternatively, if we fix $n_{s}=1.0$, then $h=0.681 \pm 0.005$ and $\sum m_{v}=0.619 \pm 0.182 \mathrm{eV}$. Or if we fix $n_{s}=0.96$ as estimated from the spectrum of CMB fluctuations, then $h=0.685 \pm 0.006$ and $\sum m_{v}=0.440 \pm 0.189 \mathrm{eV}$, see Figure B. At the Guadeloupe 2018 Conference, Adam Riess, representing the $\mathrm{SH}_{0} \mathrm{ES}$ Team, presented the latest direct measurement of the expansion parameter: $h=0.7353 \pm 0.0162$, which corresponds to negative $\sum m_{v}$ ! Discussions on these tensions made the Guadeoulpe meeting extremely interesting. And the solution may come from an unexpected direction: gravitational waves from merging black holes are a "standard siren". The single black hole merger GW170817 already obtains $h=0.70_{-0.08}^{+0.12}$, see the talk by Archil Kobakhidze!

\section{References}

[1] Lesgourgues J., and Pastor S., “Massive neutrinos and cosmology"; Phys. Rep. 429 (2006) 307

[2] Steven Weinberg, Cosmology, Oxford University Press (2008)

[3] R.K. Sachs and A.M. Wolfe, Astrophys. J. 147, 73 (1967) 


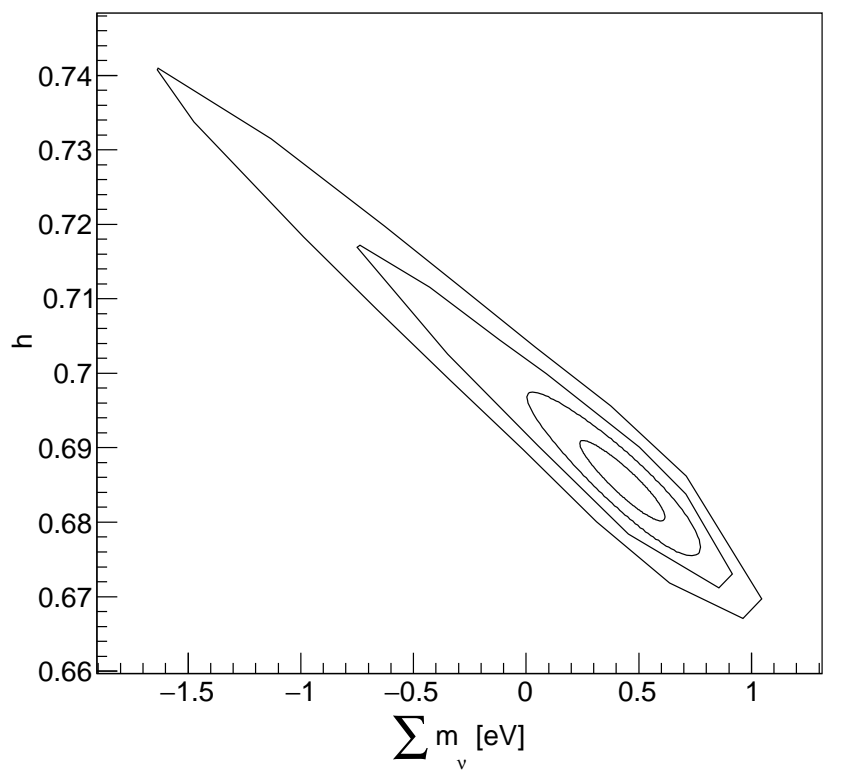

Figure 3: Contours corresponding to 1, 2, 3, and 4 standard deviations in the $\left(\sum m_{v}, h\right)$ plane, from SachsWolfe, $\sigma_{8}, 4 \sigma / \bar{N}$, and BAO measurements. $n_{s}=0.96$ is fixed (as estimated from the CMB fluctuation spectrum). Points on the contours have $\chi^{2}-\chi_{\min }^{2}=1,4,9$, and 16 , respectively, where $\chi^{2}$ has been minimized with respect to $N^{2}, b_{0}$, and $b_{s}$. The total uncertainty of $\sum m_{v}$ is dominated by the uncertainty of $h$. In this figure the systematic uncertainties presented in Equation (6.3) are not included.

[4] C. Patrignani et al., Review of Particle Physics, Chin. Phys. C, 40, 100001 (2016)

[5] Guadeloupe 2018 meeting talks: https://indico.cern.ch/event/645015/timetable/\#20180625

[6] B. Hoeneisen, Study of galaxy distributions with SDSS DR14 data and measurement of neutrino masses, arXiv:1806.01227 (2018), to be published in International Journal of Astronomy and Astrophysics

[7] L. Anderson et al., MNRAS 427, 3435 (2012).

[8] Dawson, K.S., et al., The Baryon Oscillation Spectroscopic Survey of SDSS-III, The Astronomical Journal, Volume 145, Issue 1, article id. 10, 41 pp. (2013)

[9] Blanton, M.R. et al., Sloan Digital Sky Survey IV: Mapping the Milky Way, Nearby Galaxies, and the Distant Universe, The Astronomical Journal, Volume 154, Issue 1, article id. 28, 35 pp. (2017)

[10] B. Hoeneisen, (2018) Constraints on Neutrino Masses from Baryon Acoustic Oscillation Measurements. International Journal of Astronomy and Astrophysics, 8, 1-5. https://doi.org/10.4236/ijaa.2018.81001

[11] B. Hoeneisen, (2017) Study of Baryon Acoustic Oscillations with SDSS DR13 Data and Measurements of $\Omega_{k}$ and $\Omega_{\mathrm{DE}}(a)$. International Journal of Astronomy and Astrophysics, 7, 11-27. https://doi.org/10.4236/ijaa.2017.71002 